\title{
Fundamental Limits of a Dense IoT Cell in the Uplink
}

\author{
Jean-Marie Gorce ${ }^{1}$, Yasser Fadlallah ${ }^{1}$, Jean-Marc Kelif ${ }^{2}$, H. Vincent Poor ${ }^{3}$, and Azeddine Gati ${ }^{2}$ \\ ${ }^{1}$ Univ. Lyon, INSA Lyon, Inria, CITI, F-69621 Villeurbanne, France \\ 2 Orange Labs, Issy Les Moulineaux, France \\ ${ }^{3}$ Electrical Engineering Dept. Princeton University, Princeton, NJ-08544, USA \\ \{jean-marie.gorce@insa-lyon.fr $\}$
}

\begin{abstract}
The envisioned Internet of Things (IoT) will involve a massive deployment of objects connected through wireless cells. While commercial solutions are already available, the fundamental limits of such networks in terms of node density, achievable rates or reliability are not known.

To address this question, this paper uses a large scale Multiple Access Channel (MAC) to model IoT nodes randomly distributed over the coverage area of a unique base station. The traffic is represented by an information rate spatial density $\rho(x)$. This model, referred to as the Spatial Continuum Multiple Access Channel, is defined as the asymptotic limit of a sequence of discrete MACs. The access capacity region of this channel is defined as the set of achievable information rate spatial densities achievable with vanishing transmission errors and under a sumpower constraint. Simulation results validate the model and show that this fundamental limit theoretically achievable when all nodes transmit simultaneously over an infinite time, may be reached even with a relatively small number of simultaneous transmitters (typically around 20 nodes) which gives credibility to the model. The results also highlight the potential interest of non-orthogonal transmissions for IoT uplink transmissions when compared to an ideal time sharing strategy.
\end{abstract}

\section{INTRODUCTION}

The Internet of Things (IoT) is now widely recognized as the next step of disruptive digital innovation. The IoT paradigm relies on the deployment of billions of physical things connected seamlessly to the Internet. The objective of an IoT radio access network is to provide things with an efficient worldwide real-time connection. From a technical perspective, optimizing such an architecture is challenging. As analyzed in [1] and [2], several dedicated technologies have been recently proposed, either standardized (the Lora ${ }^{\mathrm{TM}}$ consortium) or proprietary (e.g. Sigfox ${ }^{\mathrm{TM}}$ ). However, this kind of network is not a simple extension of cellular networks, due to the specific nature of the information flows. Indeed, a typical IoT cell is made of a huge number of nodes randomly transmitting small information quantities. The fundamental limits of this kind of network in terms of capacity, energy efficiency or latency have not been established yet.

The basic scenario considered in this paper includes a unique base station (BS) covering a region containing a high density of IoT nodes. For this paper, we do not consider any latency constraints and we are interested in deriving the maximal node spatial density, or more precisely, the set of information rate spatial densities $\rho(x)$, achievable under some power constraint. The objective is to determine an outer bound of the achievable rate region.

In [3], we proposed a new formalism to study dense cells. The downlink mode, usually modeled as a $k$-user Broadcast Channel (BC), led to the Spatial Continuum Broadcast Channel (SCBC) obtained as the limit of a sequence of BCs when $k \rightarrow \infty$. Its access capacity region, defined as the set of information rate spatial densities achievable under a given power, was established. This result was straightforwardly extended to the uplink by invoking the multiple access channel (MAC)-BC duality.

In the following work, we provide a formal definition of the Spatial Continuum Multiple Access Channel (SCMAC) and we derive its access capacity region when individual powers are transferable. This result relies directly on the capacity region of the $k$-user MAC.

This capacity region is known [4] and has been extensively used for cellular networks (e.g. [5], [6]) and more recently for IoT networks [7]. However, in these papers, the performance analysis is done in two steps: first, a set of nodes is randomly drawn in the cell area, and a joint transmission scheme modeled as a $K$-user MAC is optimized. Then, general performance results are obtained with Monte-Carlo simulations where the former results are averaged over a large number of random draws according to some probability law and eventually relying on the scheduler properties. Despite its interest, this approach does not lead to a fundamental limit in Shannon's sense since the achievable rates thus obtained depend on the selected scheduling protocol and the sampling time. Additionally no converse is established.

The SCMAC model herein proposed establishes a fundamental limit, independently of any scheduling protocol or time sampling.

In the following, Section II derives the SCMAC model and its properties. In Section III, the access capacity region is defined and characterized analytically. Section IV describes a simple reference scenario and presents system level simulation results validating the model.

\section{THE SCMAC MODEL}

\section{A. General assumption and definitions}

An isolated cell $\mathcal{C}$ comprises a unique BS with its service area $\Omega \subset \mathbb{R}^{2}$ and is mathematically defined by $(\Omega, \mathcal{A}, m)$ with 


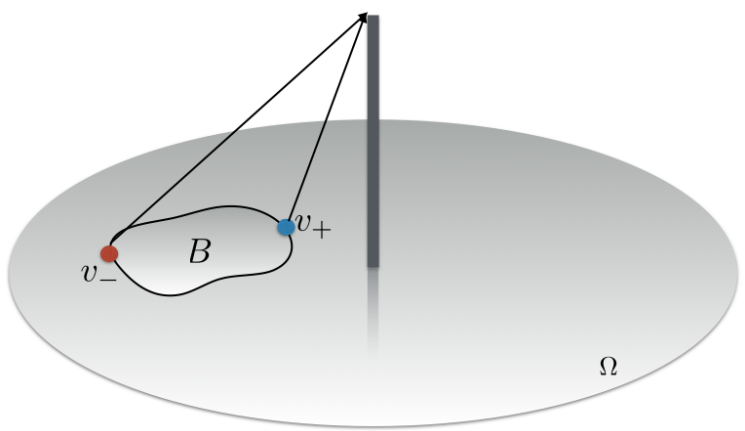

Figure 1. A PF node (Def. 2.2) is a virtual node associated with any subset $B \in \mathcal{A}$. Its rate $R(B)$ corresponds to the integration of $\rho(x)$ over $B$ (Prop. 2.1 ). The worst PF node $v_{-}(B)$ (resp. best, $v_{+}(B)$ ) associated with $B$, is the point of $B$ with the worst (resp. best) channel.

$\mathcal{A}$ the Lebesgue $\sigma$ - algebra and $m$ the Lebesgue measure. This study is restricted to Gaussian channels and single antenna transceivers.

Our objective is to establish a capacity theorem for this cell in terms of achievable spatial densities of information rates. What achievability means for a rate spatial density is not straightforward but was rigorously defined in [3] for the dual scenario (downlink). Without lack of generality, the BS is located at point $(0,0)$ and the nodes are spread over time and space on $\Omega$ according to some spatial density $u(x)$. The average number of nodes per time unit in a given subset $B \in \mathcal{A}$ is denoted by $U(B) . U(\Omega)$ is denoted by $U_{T}$ for short.

Definition 2.1 (Rate spatial density): The rate spatial density $\rho(x): \Omega \rightarrow \mathbb{R}$ is a measurable function that represents the information rate density at $x ; \forall x \in \Omega$.

$\rho(x)$ is normalized ${ }^{1}$ by the system bandwidth and is expressed in bits per channel use (bpcu) per $m^{2}$. The cell sum-rate per channel use is termed the spectral efficiency:

$$
\eta_{s}=\int_{\Omega} \rho(x) \cdot m(d x) .
$$

The uniform rate condition holds when each node requests the same quantity of information, denoted by $\mathcal{I}_{0}$, with

$$
\rho_{u}(x)=\mathcal{I}_{0} \cdot u(x) .
$$

It follows that $\eta_{s}=\mathcal{I}_{0} \cdot U_{T}$.

$\rho(x)$ is said to be achievable with a given sum-power $P$ if a transmission technique exists such that all the nodes can transmit their information with zero error probability and with a sum-power lower than $P$. Following [3], a few definitions and properties are necessary. by

Property 2.1: The rate requested by a subset $B \in \mathcal{A}$ is given

$$
R(B)=\int_{B} \rho(x) \cdot m(d x),
$$

where all information elements are assumed to be independent.

Definition 2.2 (Physically feasible (PF) node): For any subset $B \in \mathcal{A}$, a PF node $v(B)$ is defined by selecting a

\footnotetext{
${ }^{1} \rho(x)$ and related quantities $\left(\mathcal{I}_{0}, R, \ldots\right)$ are given in bits-per-channel-use (bpcu) throughout this paper.
}

point $x \in B$ with its own channel characteristics $h(x)$ and a rate equal to the sum-rate of $B$ given by (3), see Fig. 1 .

Let $\mathcal{B}=\left\{B_{k} \in \mathcal{A} ; k \in[1 ; K]\right\}$ be a partition of $\Omega$. A PF node $v_{k}$ selected according to some rule, is associated with each element $B_{k} .\left\{v_{1}, \ldots, v_{K}\right\}$ and the BS form a $K$-user MAC, denoted by $\mathfrak{N}(\mathcal{B})$, which is called a physically feasible network (PFN) that approximates the SCMAC.

The quality of a PFN approximation depends on the partition size: the larger, the better.

Definition 2.3 (Sequence of physically feasible networks): Consider a sequence of partitions $\left\{\mathcal{B}^{(i)} ; \forall i \in \mathbb{N}^{+}\right\}$with $\mathcal{B}^{(0)}=$ $\left\{B_{0}^{(0)}=\Omega\right\}$ and where a splitting process divides each set $B_{k}^{(i)}$ into two subsets $\left\{B_{2 k}^{(i+1)}, B_{2 k+1}^{(i+1)}\right\} \cdot\left\{\mathfrak{N}^{(i)}=\mathfrak{N}\left(\mathcal{B}^{(i)}\right)\right\}$ forms a PFN sequence.

\section{B. Gaussian SCMAC: definition}

Definition 2.4 (GSCMAC):

Given:

- $\Omega$, a subspace on a Hilbert space of dimension 2,

- $\mathcal{X}_{c}$, an encoding alphabet,

- $\Xi=\left\{\Phi(x): \Omega \rightarrow \mathcal{X}_{c}\right\}$, a set of fields $\Phi(\cdot)$ on $\Omega$, representing the encoded input,

- $x_{0}=(0,0) \in \Omega$, the receiver position, and

- $y \in \mathcal{Y} \subset \mathbb{R}$ the channel output observed at $x_{0}$,

the GSCMAC is a function that maps any input field $\Phi(\cdot) \in \Xi$ to a conditional probability density function (pdf) on $\mathbb{R}$ :

$$
\mathfrak{S}=\left(\Xi, Y, \mathrm{p}_{Y \mid \Xi}\right),
$$

with

$$
\mathbb{P}_{Y \mid \Xi} \sim \mathcal{N}\left(\int_{\Omega} h(x) \cdot \Phi(x) \cdot d \Omega, \sigma^{2}\right),
$$

where $h(\cdot)$ represents a linear channel and $\mathcal{N}(\mu, \nu)$ denotes a normal distribution of mean $\mu$ and variance $\nu . \sigma^{2}$ is the receiver noise variance.

In this paper, the system is constrained by the sum-power (integrated over $\Omega$ ) and not by individual powers. This model is known as the GMAC with transferable power. The capacity region of the GMAC with transferable power is known and achievable with simultaneous transmissions, a simple power allocation policy [8] and using successive interference cancellation (SIC) at the receiver, with a natural ordering of the nodes as a function of their pathloss strengths. It is worth noting that this capacity region bound is also an outer bound on the GMAC capacity region with individual power constraints [4], [8].

\section{Physically feasible networks and relative achievability}

The GSCMAC capacity region cannot be handled directly but it can be be defined at the limit of a PFN sequence.

Any given PFN $\mathfrak{N}(\mathcal{B})$ forms a GMAC for which a transmission technique can be derived.

Definition 2.5 (Relative transmission technique): A transmission technique $\left(M_{1}, \ldots, M_{K}, n, \epsilon\right)$ for $\mathfrak{N}(\mathcal{B})$ is given by $K$ individual encoders that select independently the encoded sequences $c^{n}(k)=\left[c_{1}(k), \ldots, c_{n}(k)\right]$ over $n$ channel uses, 
as a function of the individual messages $M_{k}$, and where the unique decoder observes a noisy version of the weighted sum of these symbols and decodes and estimates simultaneously the $K$ messages $\left(\hat{m}_{1}, \ldots, \hat{m}_{K}\right)$, with an error probability lower than $\epsilon$.

This paper focuses on the asymptotic regime, when $n \rightarrow \infty$ and $\epsilon \rightarrow 0$.

Definition 2.6 (Physically feasible transmitter): A PF transmitter associated with a subset $B_{k} \in \mathcal{B}$ performs two successive operations to transmit a message $M_{k}$ :

$$
M_{k} \stackrel{\mathcal{C}}{\longrightarrow} c^{n}(k) \stackrel{\mathcal{S}}{\longrightarrow} \Phi_{B_{k}}^{n}(x)=c^{n}(k) \cdot \delta\left(x_{k}\right) .
$$

After a classical encoding, a physical point $x_{k} \in B_{k}$ is selected to transmit a symbol. Therefore, the input field $\Phi$ on $B_{k}$ is equal to a delta function centered on $x_{k}$ and the global input field is

$$
\Phi^{n}(x)=\sum_{k} c^{n}(k) \cdot \delta\left(x_{k}\right) .
$$

To derive the capacity region bound, two specific selectors $\mathcal{S}$ are defined. The best selector, denoted by $\mathcal{S}_{+}$, selects the point with the best (i.e., least) pathloss, while the worst selector, denoted by $\mathcal{S}_{-}$, selects the point with the worst (i.e, highest) pathloss (see Fig. 1). For a given partition $\mathcal{B}$, the best and the worst PFNs are denoted by $\mathfrak{N}_{+}(\mathcal{B})$ and $\mathfrak{N}_{-}(\mathcal{B})$ respectively. In the following, for consistency, the subscript \pm will refer to either the worst or the best PFN.

Definition 2.7 (Relative achievability): An uplink rate density $\rho(x)$ is said to be achievable with respect to (w.r.t.) $\mathfrak{N}_{ \pm}(\mathcal{B})$, if the rate tuple $\left(R\left(B_{1}\right) \ldots R\left(B_{K}\right)\right)$ belongs to $\mathcal{C}\left(\mathfrak{N}_{ \pm}(\mathcal{B})\right)$, the capacity region of $\mathfrak{N}_{ \pm}(\mathcal{B})$.

We now have all the tools to derive the main theoretical results.

Theorem 2.1 (Relative achievability with worst selectors implies asymptotic achievability): Consider a sequence of worst PFNs denoted by $\left\{\mathfrak{N}_{-}^{(i)}\right\}$. If $\rho(x)$ is achievable w.r.t. $\mathfrak{N}_{-}^{(i)}$ for some $i \geq 0$, then $\rho(x)$ is also achievable w.r.t. $\mathfrak{N}_{-}^{(j)}$, $\forall j \geq i$. Thus, the capacity regions satisfy

$$
\mathcal{C}\left(\mathfrak{N}_{-}^{(i)}\right) \subset \mathcal{C}\left(\mathfrak{N}_{-}^{(j)}\right) ; \forall j \geq i .
$$

Therefore, if $\rho(x)$ is achievable w.r.t. the worst PFN associated with any partition $\mathcal{B}$, then $\rho(x)$ is asymptotically (when $i \rightarrow$ $\infty)$ achievable.

Proof: The proof relies on time-sharing. Consider a subset $B_{k}^{(i)}$ and its worst node $v_{-, k}$. When the subset is split into two subsets $B_{m}^{(i+1)}$ and $B_{n}^{(i+1)}$, the former keeps the same node $v_{-, n}=v_{-, k}$ while the latter selects its worst node that has a better channel than $v_{-, k}$. Since $v_{-, k}$ is able to transmit at rate $R\left(B_{k}\right)=R\left(B_{n}\right)+R\left(B_{m}\right)$, a simple time sharing between $v_{-, n}$ and $v_{-, m}$ achieves the desired rates.

Theorem 2.2 (Converse: relative non-achievability with best selectors implies asymptotic non-achievability): Consider a sequence of best PFNs denoted by $\left(\mathfrak{N}_{+}^{(i)}\right)$. If $\rho(x)$ is not achievable w.r.t. $\mathfrak{N}_{+}^{(i)}$ for some $i \geq 0$, then $\rho(x)$ is also not achievable w.r.t. $\mathfrak{N}_{+}^{(j)}, \forall j \geq i$ :

$$
\mathcal{C}\left(\mathfrak{N}_{+}^{(i)}\right) \supset \mathcal{C}\left(\mathfrak{N}_{+}^{(j)}\right) ; \forall j \geq i
$$

Therefore, if $\rho(x)$ is not achievable w.r.t. the best PFN associated with any partition $\mathcal{B}$, then $\rho(x)$ is asymptotically not achievable.

Proof: Consider a subset $B_{k}^{(i)}$ with its own best transmitter $v_{+, k}$. When the subset is split into two subsets $B_{n}^{(i+1)}$ and $B_{m}^{(i+1)}$, one subset keeps the same transmitter $v_{+, n}=v_{+, k}$ while the other subset selects another transmitter $v_{+, m}$ with a worse channel. Let us assume that a transmission scheme exists with the partition $(i+1)$ such that the desired rates $R\left(B_{n}\right)$ and $R\left(B_{m}\right)$ are achievable. Then, $v_{+, k}$ is obviously able to transmit at the sum-rate $R\left(B_{k}\right)=R\left(B_{n}\right)+R\left(B_{m}\right)$.

\section{GSCMAC ACCESS CAPACITY REGION}

The access capacity region ${ }^{2}$ is defined as the set of all rate spatial densities $\rho(x)$ that are asymptotically achievable with a given sum-power $P_{m}$. To characterize this set, we use a specific PFN sequence that lets the capacity regions of the worst and best PFNs converge to the same limit. This limit is called the doubly asymptotic access capacity region of the GSCMAC $\mathfrak{S}$. The term doubly asymptotic refers to the fact that this capacity is defined for $n, i \rightarrow \infty$.

The access capacity region derives straightforwardly from the asymptotic achievability stated in Theorems 2.1 and 2.2.

Definition 3.1 (Access capacity region): The access capacity region noted $\mathcal{D}_{\mathfrak{S}}$ is the set of asymptotically achievable spatial densities $\rho(x)$.

Theorems 2.1 and 2.2 provide upper and lower bounds of the asymptotic capacity region. Clearly, the capacity regions associated with a sequence of partitions satisfy

$$
\begin{aligned}
& \mathcal{C}\left(\mathfrak{N}_{-}^{(0)}\right) \subset \mathcal{C}\left(\mathfrak{N}_{-}^{(1)}\right) \subset \cdots \subset \mathcal{C}\left(\mathfrak{N}_{-}^{(\infty)}\right) \\
& \mathcal{C}\left(\mathfrak{N}_{+}^{(0)}\right) \supset \mathcal{C}\left(\mathfrak{N}_{+}^{(1)}\right) \supset \cdots \supset \mathcal{C}\left(\mathfrak{N}_{+}^{(\infty)}\right) .
\end{aligned}
$$

The key point is to chose a splitting process such that

$$
\lim _{i \rightarrow \infty} \mathcal{C}\left(\mathfrak{N}_{+}^{(\infty)}\right)=\lim _{i \rightarrow \infty} \mathcal{C}\left(\mathfrak{N}_{-}^{(\infty)}\right)
$$

If it exists, this limit is unique and is equal to $\mathcal{D}_{\Omega}$. Basically, the worst PFN sequence provides successive achievable regions while the best $\mathrm{PFN}$ sequence provides successive converse regions.

Let us decompose $\rho(x)$ as $\rho(x)=\eta_{s} \cdot f_{\rho}(x)$ where $f_{\rho}(x)$ is a normalized rate distribution $\left(\int_{\Omega} f_{\rho}(x) \cdot m(d x)=1\right)$. Then the access capacity region is alternatively characterized: for any distribution $f_{\rho}(x)$ (integrable, positive definite), the capacity region bound is reached by choosing the maximal value of $\eta_{s}$ such that $\rho(x) \in \mathcal{D}_{\Omega}$. Then, the access capacity region is computed under its dual form:

Definition 3.2 (Dual): Consider a rate distribution $\rho(x)$, with a sum-rate $\eta_{S}$. The minimal power $\tilde{P}_{m}$ required to ensure the asymptotic achievability is given by

$$
\tilde{P}_{m}=\min _{P \in \mathbb{R}^{+}} P=\int_{\Omega} d P(x) \quad \text { s.t. } \quad \eta_{S} \cdot f_{\rho}(.) \in \mathcal{D}_{\mathfrak{S}}(P),
$$

${ }^{2}$ We use the term access capacity region to avoid confusion with the classical MAC capacity that is expressed as a set of joint rates, while here the access capacity region is defined as a set of information rate spatial densities. 
where $\mathcal{D}_{\mathfrak{S}}(P)$ is the access capacity region of $\mathfrak{S}$ with sumpower $P$.

Let us define $\nu(x)$, the equivalent noise at the receiver, for a transmitter located in $x$, i.e. $\nu(x)=\sigma^{2} / g(x)$ with $g(x)=$ $|h(x)|^{2}$. The complementary cumulative distribution function (ccdf) of the nodes' equivalent noise power is defined as

$$
G_{\nu}(\nu)=\int_{\Omega} f_{\rho}(x) \cdot \mathbb{1}[\nu(x) \geq \nu] \cdot m(d x),
$$

and represents the sum-rate proportion associated with transmitters with an equivalent noise larger than $\nu$. The corresponding pdf is $f_{\nu}(\nu)=-d G_{\nu}(\nu) / d \nu$.

Theorem 3.1 (GSCMAC minimal sum-power): The minimal sum-power required to achieve a given rate distribution $\rho(x)$ in uplink mode is

$$
\tilde{P}_{m}=a \cdot \eta_{s} \int_{\nu_{m}}^{\nu_{M}} t \cdot f_{\nu}(t) \cdot e^{a \cdot \eta_{s} \cdot G_{\nu}(t)} \cdot d t,
$$

with $a=2 \log (2)$.

Proof: The detailed proof given in the appendix uses a specific PFN sequence and establishes a differential equation representing the sum-power as a function of the cell load.

This result is the main theoretical result of this paper, which completes the derivation proposed for the GSCBC in [3]. Additionally, it proves that the BC-MAC duality [9] extends to the GSCBC-GSCMAC.

\section{VALIDATION ON A REFERENCE SCENARIO}

The interest of the proposed formalism is now illustrated on a simple scenario. It is worth noting that the model could be extended to more complex scenarios with multiple cells and shadowing. For a multicell extension, the reader is referred to [10]. Alternatively, the strategy used in [5] where the interference from neighboring cells is considered as a whole with a weighting factor could be used.

\section{A. GSCMAC fundamental limit}

A unique cell covering a disk of radius $R$ is considered, in the uplink. For the sake of simplicity, a simple power-law pathloss and omnidirectional antennas are considered with no shadowing:

$$
g(x)=g_{0} \cdot|x|^{-\alpha},
$$

where $g_{0}$ and $\alpha$ represent respectively a constant and the attenuation slope. The transmission sum-power constraint is given by $\sum_{k} P_{k} \leq P_{M}$.

For the sake of simplicity, the fundamental limit is studied for a uniform rate density, $\rho(x)=\rho_{0}$, which means that $\eta_{S}=\pi \rho_{0} R^{2}$ and $f_{\rho}(x)=1 /\left(\pi R^{2}\right) . \eta_{s}$ represents the uniform capacity of this cell and the fundamental limit is characterized by the minimal sum-power allowing support of the rate density $\rho(x)$.

With this model, the equivalent noise distribution straightforwardly derives as (see [3] for details)

$$
f_{\nu}(\nu)=\frac{2}{\alpha \cdot \nu_{R}} \cdot\left(\frac{\nu}{\nu_{R}}\right)^{2 / \alpha-1}, \quad \forall \nu \in\left[0 ; \nu_{R}\right]
$$

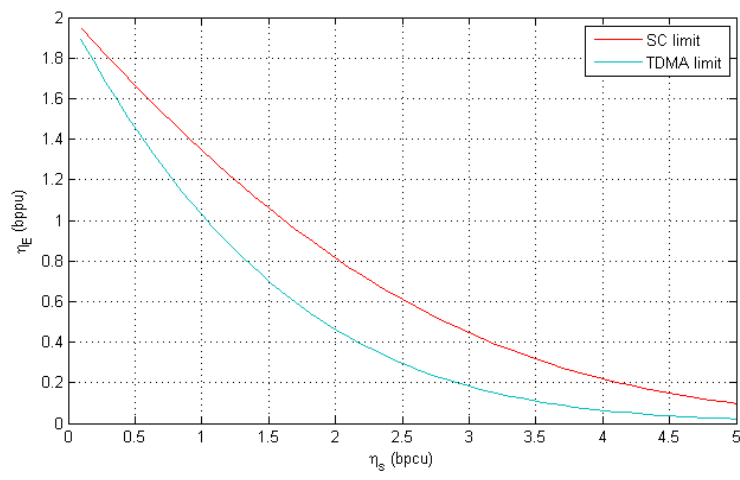

Figure 2. The EE-SE achievable region bound is achieved with SC (red curve). Achievability with TDMA is drawn in light blue for comparison purpose.

Using (12) in (10), one obtains

$$
\tilde{P}_{m}=\nu(R) \cdot\left(a \eta_{s}\right)^{-\alpha / 2} \cdot e^{a \eta_{s}} \cdot \Gamma\left(1+\frac{\alpha}{2}, a \eta_{s}\right),
$$

with $\Gamma(a, x)$ the incomplete gamma function. Eq. (13) provides the energy efficiency - spectral efficiency (EE-SE) fundamental limit of the cell. Alternatively, the inverse of this equation characterizes the cell uniform capacity by providing $\eta_{S}$ as a function of $P_{m}$. For consistency, the power is normalized by the equivalent noise received from a transmitter located at the cell edge $\left(p_{r}=P_{M} / \nu(R)\right)$ and the EE in bits per relative power use (bppu) is defined as $\eta_{E}=\eta_{S} / p_{r}$ leading to

$$
\eta_{E}=\frac{1+\alpha / 2}{a \cdot{ }_{1} F_{1}\left(1 ; 2+\alpha / 2 ; a \eta_{S}\right)} .
$$

The EE-SE achievable region is represented in Fig. 2 with $\alpha=3.65$ and $g_{0} / \sigma^{2}=1$. The red curve shows the fundamental limit of the uniform capacity region in terms of EE-SE. Any point of this limit is achievable with an $\infty$-user superposition coding (SC) strategy with an optimal SIC receiver. For comparison purposes, the region achievable with pure orthogonal sharing is also represented and tagged as Time Division Multiple Access (TDMA).

In the low spectral efficiency regime, when $\eta_{S} \rightarrow 0$, TDMA and SC converge to the same limit known as the wideband limit [11]. But, in the high SE regime, the TDMA performance goes away from the fundamental limit.

\section{B. Simulation Results}

In the previous section, we derived an analytical expression for the EE-SE fundamental limit of an isolated cell. The theory developed in this paper proves that the fundamental limit can be reached with an SC/SIC strategy (see the appendix) in which all nodes randomly deployed according to the random process $u(x)$ have to transmit simultaneously their own packet spread over an infinite time. At this point we may wonder if this fundamental limit can be reached with a more feasible algorithm in which the SC/SIC strategy would be used by successive groups of nodes. This scheme would be a mix of TDMA and SC. For the sake of simplicity, we consider an ideal system with perfect channel state information at the transmitters (CSIT), and we consider that a sub-group of nodes 


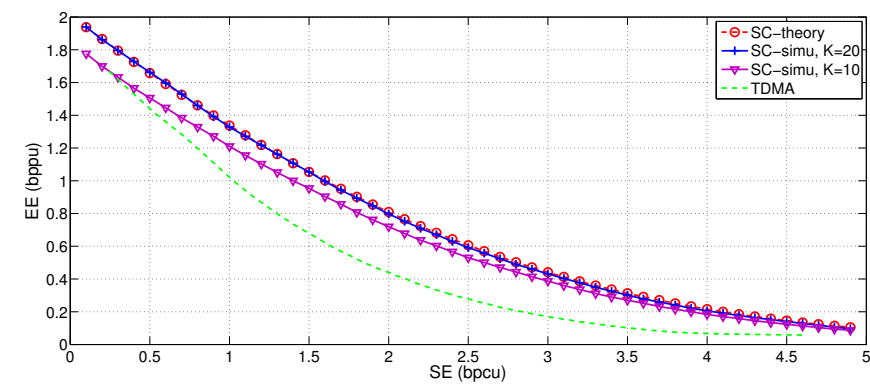

Figure 3. Comparison of the theoretical achievable limits versus simulated EE-SE performance using superposition coding for $K=10$ and $K=20$.

randomly selected are transmitting simultaneously with an optimal power allocation.

\section{Simulation parameters}

The parameters of the system level simulator are given in Table I.

\begin{tabular}{|c|c|}
\hline Cell layout & One circular cell model \\
\hline Cell diameter & $500 \mathrm{~m}$ \\
\hline Node distribution & Uniform \\
\hline Minimum node distance & $35 \mathrm{~m}$ \\
\hline Number of bits & 100 \\
\hline Antenna type & Omni-directional \\
\hline Transmission mode & Uplink single-input-single-output \\
\hline Pathloss model & $-36.5 \log _{10}(r) \mathrm{dB}$ \\
\hline Noise density power & $-166 \mathrm{dBm} / \mathrm{Hz}$ \\
\hline Scheduling & SC: Simultaneous transmission \\
\cline { 2 - 2 } & TDMA: Time division slots \\
\hline & Table I \\
&
\end{tabular}

For the sake of compatibility with our theoretical analysis, we do not assume any fading. A large set of nodes is initially uniformly distributed in the cell. Let us denote a subset of nodes $\left\{u_{1}, u_{2}, \cdots, u_{k}\right\}$ with channel gains $g_{1}<g_{2}<\cdots<$ $g_{k}$, randomly drawn. Therefore, for a maximum sum-power efficiency, the power is distributed such that $u_{K}$ is decoded first while $u_{1}$ is decoded last, which means that the $k^{t h}$ node transmits with a power $P_{k}$ given by

$$
P_{k}=\frac{N_{0}+\sum_{i=1}^{k-1} g_{i} P_{i}}{g_{k}}\left(2^{2 \eta_{s}(k)}-1\right),
$$

where $\eta_{s}(k)$ is the targeted spectral efficiency for user $k$. Since a uniform rate distribution is assumed, we have $\eta_{s}(k)=\eta_{s} / K$. A classical SIC receiver is employed at the BS.

\section{Results}

Monte-Carlo simulations are run for $10^{5}$ independent scenarios with different power levels. During one scenario, each node transmits 100 bits of data. When TDMA is considered, the power transmission level is kept constant for all users, but the time-slot length is adapted as a function of the pathloss coefficient, such that each node may transmit all of its information without error. This scheme is slightly sub-optimal since the power levels are not adapted as a function of the channel gain but the efficiency loss is limited [10].

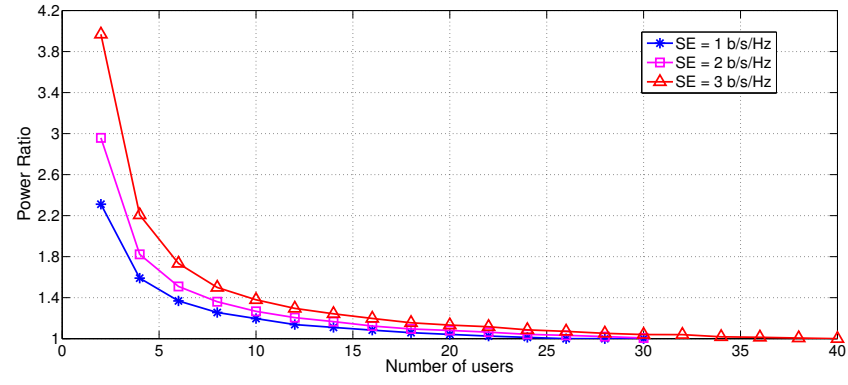

Figure 4. Convergence of the simulated optimal power allocation to the theoretical limits, with respect to the number of nodes in the cell.

Using SC, all nodes transmit over all time slots simultaneously, while individual powers are calculated based on (15) to obtain perfect reception with the SIC receiver. Fig. 3 compares the fundamental limit (EE-SE) of three scenarios: a reference orthogonal resource sharing (TDMA), and two SC strategies with $K=10$ and $K=20$.

This figure shows that for this scenario, $K=20$ is enough to reach the fundamental limit while SC with $K=10$ induces EE loss of about $10 \%$ in the low SE regime. Both SC schemes outperform TDMA. This figure shows an excellent agreement between the fundamental limit and the simulated results even with finite sets of nodes.

To evaluate the impact of the number of simultaneous transmitting nodes, the ratio between simulated average power and the theoretical limit (10), given by $\mathbb{E}\left[P_{t}\right] / \tilde{P}_{m}$, is shown in Fig. 4 w.r.t. the number of simultaneous transmitting nodes $K$. To provide a significant gain, $\mathrm{SC}$ requires enough simultaneous transmissions (greater than 5). The convergence w.r.t. $K$ is faster for smaller $\eta_{S}$.

In addition, Fig. 5 illustrates the power distribution as a function of the BS-node distance in a 20-node scenario and for a target $\eta=2 \mathrm{bpcu}$. Note that the SC curve is mathematically modeled by (23). Interestingly, with SC, the maximal power is not obtained at the cell edge. This is due to the SC algorithm which favors edge users.

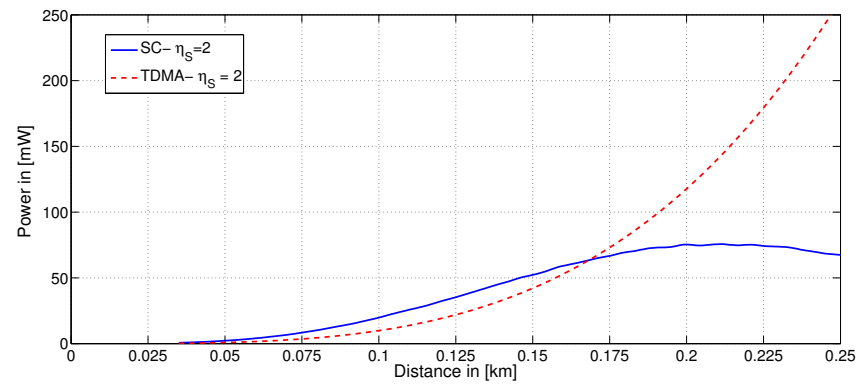

Figure 5. The power distribution as a function of the distance between the nodes and the BS using TDMA and SC for $K=20$ and $\eta_{S}=2$.

\section{CONCLuSion}

In this paper, we have extended the spatial continuum approach proposed in [3] to the SCMAC. We have derived 
the analytical expression for the access capacity region. We have applied the model in a simple scenario and the simulation results show that the fundamental limit can be almost reach with an SC strategy, even with a limited number of nodes transmitting simultaneously $(\approx 20)$.

This model provides interesting insights for future IoT network optimization. We have observed for instance how SC allows a reduction in the transmission power of edge nodes which may bring strong benefits to the network performance: e.g. by increasing the network life time by preserving the power of edge nodes, or by reducing inter-cell interference.

\section{APPENDIX: PROOF OF THEOREM 3.1}

The proof exploits Theorems 2.1 and 2.2 with a specific PFN sequence $\mathfrak{N}^{(i)}$. We first apply these theorems to $B_{0}^{(0)}=$ $\Omega$. The sum-rate $\eta_{S}$ is bounded by the point-to-point capacity with either the best or the worst transmitters:

$$
\log \left(1+\frac{\tilde{P}_{m}}{\nu_{-}}\right) \leq a \cdot \eta_{S} \leq \log \left(1+\frac{\tilde{P}_{m}}{\nu_{+}}\right)
$$

Given $B_{k}^{(i)}$ with $\nu(x) \in\left[\nu_{m, k}^{(i)}, \nu_{M, k}^{(i)}\right) ; \forall x \in B_{k}^{(i)}$. Then, $B_{k}^{(i)}$ is split such that

$$
\begin{aligned}
& B_{2 k}^{(i+1)}=\left\{x \in B_{k}^{(i)} ; \nu(x)<\bar{\nu}_{k}^{(i)}\right\} \\
& B_{2 k+1}^{(i+1)}=\left\{x \in B_{k}^{(i)} ; \nu(x) \geq \bar{\nu}_{k}^{(i)}\right\},
\end{aligned}
$$

where $\bar{\nu}_{k}^{(i)}=\left(\nu_{m, k}^{(i)}+\nu_{M, k}^{(i)}\right) / 2$.

The rate associated with $B_{k}^{(i)}$ is

$$
R_{k}^{(i)}=\eta_{S} \cdot \int_{\nu_{m, k}^{(i)}}^{\nu_{M, k}^{(i)}} f_{\nu}(\nu) \cdot d \nu
$$

According to Theorems 2.1 and 2.2, the access capacity region $\mathcal{D}_{\Omega}$ is outer and inner bounded by the access capacity region of the PF networks: $\mathcal{C}\left(\mathfrak{N}_{-}\right) \leq \mathcal{D}_{\Omega} \leq \mathcal{C}\left(\mathfrak{N}_{+}\right)$. The capacity of a $K$-user MAC with transferable power is known and is achieved with SIC decoding.

Thanks to the splitting process defined above, the min/max equivalent noise values associated with each subset are ordered and satisfy

$$
\nu_{m, 0}^{(i)}<\nu_{m, 1}^{(i)}<\cdots<\nu_{m, 2^{i}-1}^{(i)} \quad \text { with } \quad \nu_{M, k}^{(i)}=\nu_{m, k+1}^{(i)} .
$$

Therefore, $\tilde{P}_{m}$ is upper (resp. lower) bounded by the minimal power required to serve the worst (resp. the best) PFN. The minimal transmission power required for each $\mathrm{PF}$ node $v_{k}$ is known for an SC/SIC transmission and follows:

$$
g_{ \pm, k}^{(i)} \cdot P_{ \pm, k}^{(i)}=\left(e^{a R_{k}^{(i)}}-1\right) \cdot\left(N_{0}+\sum_{q>k} g_{ \pm, q}^{(i)} \cdot P_{ \pm, q}^{(i)}\right)
$$

Let the power received at the BS be denoted by $Q_{ \pm, k}^{(i)}=$ $g_{ \pm, k}^{(i)} \cdot P_{ \pm, k}^{(i)}$. Then (18) becomes

$$
Q_{ \pm, k}^{(i)}=\left(e^{a R_{k}^{(i)}}-1\right) \cdot\left(N_{0}+\sum_{q>k} Q_{ \pm, q}^{(i)}\right)
$$

The cumulative received sum-power (for nodes $l>k$ ) is denoted by $\Pi_{ \pm, k}^{(i)}$ and is given by the recursive sum of (19):

$$
\Pi_{ \pm, l}^{(i)}=\sum_{k \geq l}\left(2^{2 R_{k}^{(i)}}-1\right) \cdot\left(N_{0}+\Pi_{ \pm, k-1}^{(i)}\right) .
$$

When $i \rightarrow \infty, \nu_{M, k}^{(i)}-\nu_{m, k}^{(i)} \rightarrow 0$; thus $\Pi_{+, k}^{(i)}-\Pi_{-, k}^{(i)} \rightarrow 0, \forall k$.

Furthermore, when $i \rightarrow \infty$, the sum-rate of each subset tends to 0 . It follows that $\left(e^{a R_{k}^{(i)}}-1\right) \rightarrow a \cdot R_{k}^{(i)}$. Using these limits in (20), $\Pi(\nu)$ becomes asymptotically the solution of the following Riemann integral:

$$
\Pi(\nu)=a \eta_{s} \cdot \int_{\nu}^{\nu_{M}} f_{\nu}(x) \cdot\left(N_{0}+\Pi(x)\right) \cdot d x .
$$

Straightforward computations lead to

$$
\Pi(\nu)=N_{0} \cdot\left(e^{a \eta_{S} \cdot G_{\nu}(\nu)}-1\right) .
$$

The transmission power density as a function of $\nu$ is obtained by deriving (22) and using $Q_{r}(\nu)=g(x) \cdot P_{t}(x)$ :

$$
P_{t}(\nu)=\nu \cdot a \eta_{s} f_{\nu}(\nu) \cdot e^{a \eta_{s} \cdot G_{\nu}(\nu(x))} .
$$

Its integration w.r.t $\nu$ leads to (10).

\section{ACKNOWLEDGEMENT}

The work of J.-M. Gorce was supported by Orange Labs under Grant no F05151 and by the French National Research Agency project ARBURST. The work of Y. Fadlallah was supported by the French government FUI project SMACS. The work of H.V. Poor was supported by the U.S. National Science Foundation Grant ECCS-1647198.

\section{REFERENCES}

[1] C. Goursaud and J.-M. Gorce, "Dedicated networks for IoT: PHY/MAC state of the art and challenges," EAI Endorsed Transactions on Internet of Things, vol. 15, 102015.

[2] M. Centenaro, L. Vangelista, A. Zanella, and M. Zorzi, "Long-range communications in unlicensed bands: The rising stars in the IoT and smart city scenarios," IEEE Wireless Commun., vol. 23, no. 5, pp. 6067, 2016.

[3] J.-M. Gorce, J.-M. Kelif, and H. V. Poor, "Spatial continuum model: Toward the fundamental limits of dense wireless networks," in Proc. IEEE Global Commun. Conf. (GLOBECOM), 2016.

[4] T. M. Cover and J. A. Thomas, Elements of Information Theory. John Wiley \& Sons, 2012.

[5] A. D. Wyner, "Shannon-theoretic approach to a Gaussian cellular multiple-access channel," IEEE Trans. Inf. Theory, vol. 40, no. 6, pp. 1713-1727, 1994.

[6] V. Chandrasekhar and J. G. Andrews, "Uplink capacity and interference avoidance for two-tier femtocell networks," IEEE Trans. Wireless Commun., vol. 8, no. 7, pp. 3498-3509, 2009.

[7] H. S. Dhillon, H. Huang, H. Viswanathan, and R. A. Valenzuela, "Fundamentals of throughput maximization with random arrivals for M2M communications," IEEE Trans. Commun., vol. 62, no. 11, pp. 40944109, 2014.

[8] D. N. Tse and S. V. Hanly, "Multiaccess fading channels. i. Polymatroid structure, optimal resource allocation and throughput capacities," IEEE Trans. Inf. Theory, vol. 44, no. 7, pp. 2796-2815, 1998.

[9] N. Jindal, S. Vishwanath, and A. Goldsmith, "On the duality of gaussian multiple-access and broadcast channels," IEEE Trans. Inf. Theory, vol. 50, no. 5, pp. 768-783, 2004.

[10] J.-M. Gorce, D. Tsilimantos, P. Ferrand, and H. V. Poor, "Energycapacity trade-off bounds in a downlink typical cell," in Proc. IEEE Int. Symp. on Personal, Indoor and Mobile Radio Commun.(PIMRC), 2014.

[11] S. Verdú, "Spectral efficiency in the wideband regime," IEEE Trans. Inf. Theory, vol. 48, no. 6, pp. 1319-1343, 2002. 